\title{
Síndrome de Liddle: diagnóstico tardio de causa rara de hipertensão arterial
}

\author{
Liddle's syndrome: late diagnosis of a rare cause of arterial hypertension
}

\author{
Ana Cristina Simões e Silva ${ }^{1}$, Eduardo A. Oliveira ${ }^{1}$, Camila Romano Gomes ${ }^{2}$, \\ Flávio Souza Lima ${ }^{3}$, José S.S. Diniz ${ }^{4}$
}

\begin{abstract}
Resumo
Objetivo: a finalidade desse relato de caso é chamar a atenção para uma doença que, embora rara, é causa de hipertensão arterial grave em crianças. O relato procura chamar a atenção dos pediatras para a importância do diagnóstico precoce de hipertensão arterial, e do valor de se estabelecer uma investigação apropriada a cada caso.

Relato de caso: neste artigo é relatado uma caso de um paciente de treze anos, do sexo masculino, com quadro clínico e laboratorial típico dessa doença. O diagnóstico foi estabelecido a partir do quadro clínico associado à presença de hipocalemia crônica, aumento da excreção urinária de potássio acoplado à retenção de sódio, e diminuição da atividade de renina plasmática e dos níveis circulantes de Angiotensina II e aldosterona. Apesar da resposta inicial ao uso de triamtereno, o paciente evoluiu com perda da função renal, secundária ao retardo no diagnóstico e ao mau controle da hipertensão.

Discussão: a Síndrome de Liddle consiste numa forma de pseudo-aldosteronismo caracterizado por hipertensão arterial, hipocalemia, alcalose metabólica e retardo no crescimento. Alguns aspectos da fisiopatologia, do diagnóstico e do tratamento são discutidos no estudo.
\end{abstract}

J Pediatr (Rio J) 2002; 78 (3): 251-54: síndrome de Liddle, hipertensão arterial, insuficiência renal crônica.

\section{Introdução}

Em 1963, Liddle e colaboradores descreveram uma doença renal de caráter familiar, com características clínicas e laboratoriais semelhantes ao hiperaldosteronismo primário, porém com liberação de aldosterona em concen-

1. Professor-Adjunto, Doutor. Departamento de Pediatria - Universidade Federal de Minas Gerais (UFMG).

2. Residente de Pediatria - Hospital das Clínicas - UFMG.

3. Acadêmico estagiário da Unidade de Nefrologia - Hospital das Clínicas - UFMG.

4. Professor Emérito, Livre-Docente. Departamento de Pediatria - UFMG.

Artigo submetido em 03.11.01, aceito em 27.03.02.

\begin{abstract}
Objective: the aim of this article is to highlight the importance of a rare disease that causes severe arterial hypertension in children. It is important to advise pediatricians to measure arterial pressure in children in order to avoid late diagnosis and renal insufficiency.

Description: we report a case of a 13-year-old patient that presented typical clinical and laboratorial features of Liddle's syndrome. The diagnosis was established based on the clinical picture associated with the presence of chronic hypokalemia, increase of urinary potassium excretion with sodium retention and reduction of plasma renin activity, and circulating levels of angiotensin II and aldosterone. In a spite of the initial therapeutic response to triamterene, the patient developed progressive renal failure due to the delay in the diagnosis and the poorly controlled hypertension.

Comments: Liddle's syndrome consists of a form of pseudoaldosteronism characterized by arterial hypertension, hypokalemia, metabolic alkalosis and failure to thrive. Some aspects regarding physiopathology, diagnosis and treatment are discussed.
\end{abstract}

J Pediatr (Rio J) 2002; 78 (3): 251-54: Liddle's syndrome, arterial hypertension, chronic renal insufficiency.

trações mínimas ${ }^{1}$. O quadro caracterizava-se por hipertensão severa, expansão do volume extracelular e hipocalemia. $\mathrm{Na}$ ocasião, Liddle postulou que as alterações observadas seriam devidas a uma anormalidade primária no transporte renal de sódio, com aumento da permeabilidade a esse íon, e conseqüentes hipervolemia e perda de potássio. Atualmente, a síndrome é considerada uma doença autossômica dominante, com expressão clínica variável, cuja alteração principal ocorre nos canais de sódio, e é secundária a mutações em suas subunidades beta ou gama ${ }^{2}$.

Deve-se suspeitar desse diagnóstico em pacientes hipertensos de difícil controle, com poliúria e polidipsia, 
história familiar positiva, que exibem alcalose hipocalêmica, hiporreninemia, cortisol normal e níveis insignificantes de aldosterona ${ }^{3}$. Após confirmação, recomenda-se o tratamento com triamtereno ou amilorida, que atuam diminuindo a permeabilidade da célula tubular ao sódio através da inibição de canais amilorida-sensíveis ${ }^{4,5}$.

O diagnóstico de Síndrome de Liddle traz implicações importantes, sobretudo quando é tardio ou não é feito. Vale ressaltar ainda que, por se tratar de uma doença de caráter familiar, um aconselhamento genético, além da pesquisa nos demais membros da família, é essencial. Podem ser observadas seqüelas renais, cardiovasculares e de crescimento, sendo que, com o tratamento correto, normalmente ocorre melhora significativa do quadro clínico e laboratorial. Dessa forma, o objetivo desse relato de caso é chamar a atenção para uma doença que, embora rara, é causa de hipertensão arterial grave em crianças.

\section{Relato de caso}

Paciente FJTS, 13 anos, do sexo masculino, foi admitido no Serviço de Nefrologia Pediátrica com queixa de atraso no crescimento pôndero-estatural. A mãe relatava que, desde o nascimento, em fevereiro de 1984, a criança apresentava poliúria, polidipsia, episódios febris de repetição e retardo no crescimento, com peso e estatura constantemente abaixo do percentil 3, tendo sido tratado com polivitamínicos sem sucesso. Em relação à gestação e ao parto, a mãe apresentou hemorragia no sexto mês, tendo o menino nascido prematuro, com peso de 1.480 gramas e comprimento de $36 \mathrm{~cm}$, permanecendo em unidade de tratamento intensivo durante três meses. Não houve relato de medida da pressão arterial durante sua permanência em unidade de terapia intensiva. Houve também um diagnóstico presuntivo, não confirmado, de toxoplasmose congênita, tendo a criança recebido tratamento por tempo prolongado. $\mathrm{Na}$ história familiar, a informante relatou que o paciente tinha três irmãos mais velhos do sexo masculino, hígidos, embora a mãe tenha tido três abortos sem causa definida.
À admissão aos 13 anos, além da baixa estatura $(123,5 \mathrm{~cm}$ - percentil < 3), encontrava-se assintomático, mas com pressão arterial de 220 x $160 \mathrm{mmHg}$. A mãe relatava que a pressão arterial do paciente nunca havia sido aferida anteriormente, mesmo a criança tendo sido atendida por vários pediatras e endocrinologistas (devido à baixa estatura). Foi então optado por internação hospitalar, tendo sido iniciado nifedipina de liberação lenta e captopril, com controle parcial dos níveis pressóricos. Foi realizada propedêutica para hipertensão arterial que revelou, como alterações bioquímicas, apenas hipocalemia e as escórias no limite superior da normalidade, além de aumento de área cardíaca (RX de tórax), sobrecarga de ventrículo esquerdo (ECG) associada à hipertrofia e insuficiência aórtica leve (ecocardiograma). Ao exame de fundo de olho, detectou-se retinopatia hipertensiva e, no ultra-som abdominal, rins com aumento difuso da ecogenicidade e perda da diferenciação córtico-medular. As dimensões longitudinal, transversal e ântero-posterior dos rins foram as seguintes: direito $(73 \times 30 \times 27 \mathrm{~mm})$ e esquerdo $(77 \times 33 \times 38 \mathrm{~mm})$.

Após controle parcial dos níveis pressóricos, o paciente recebeu alta para controle ambulatorial em uso de nifedipina e captopril. A propedêutica foi ampliada em nível ambulatorial. O ultra-som Doppler dos vasos renais não demonstrou anormalidades. As determinações de eletrólitos urinários, atividade de renina plasmática (ARP) e níveis circulantes de angiotensinas [angiotensina I - Ang I, angiotensina II - Ang II e angiotensina-(1-7) - Ang-(1-7)] e aldosterona mostraram, respectivamente, aumento da excreção urinária de potássio acoplada à redução da perda de sódio, além de diminuição da ARP, dos níveis circulantes de Ang II e da aldosterona sérica (vide Tabela 1). Diante deste quadro clínico e das alterações laboratoriais, foi levantada a hipótese de Síndrome de Liddle, sendo substituído o esquema terapêutico por uma associação fixa de triamtereno ( $150 \mathrm{mg} /$ dia $)$ e hidroclorotiazida $(150 \mathrm{mg} / \mathrm{dia})$ e também cloreto de potássio $(2,4 \mathrm{~g} / \mathrm{dia})$, com boa resposta terapêutica. A pressão arterial atingiu nível normal para a idade $(120 \times 85 \mathrm{mmHg}$, percentil $<90$ para sexo e estatura).

Tabela 1 - Determinação dos componentes circulantes do sistema renina-angiotensina-aldosterona ao diagnóstico

\begin{tabular}{lc}
\hline Valores de referência & Valores determinados \\
\hline ARP (VR: $0,40 \pm 0,22 \mathrm{ng} \mathrm{Ang} \mathrm{I/} \mathrm{ml/} \mathrm{h)}$ & $0,08 \mathrm{ng}$ Ang I/ ml/h \\
Ang I (VR: $26,4 \pm 13,7 \mathrm{pg} / \mathrm{ml})$ & $27,8 \mathrm{pg} / \mathrm{ml}$ \\
Ang II (VR: $21,4 \pm 8,7 \mathrm{pg} / \mathrm{ml})$ & $16,2 \mathrm{pg} / \mathrm{ml}$ \\
Ang-(1-7) (VR: $16,1 \pm 7,9 \mathrm{pg} / \mathrm{ml})$ & $15,5 \mathrm{pg} / \mathrm{ml}$ \\
Aldosterona (VR: $5-30 \mathrm{ng} / \mathrm{dl})$ & $4 \mathrm{ng} / \mathrm{dl}$ \\
\hline
\end{tabular}


Nos controles subseqüentes, observou-se redução da poliúria e da polidipsia associada à estabilização da pressão arterial e com os níveis de potássio e escórias nitrogenadas mantendo-se estáveis.

O paciente permaneceu, então, sem comparecer aos controles ambulatoriais por aproximadamente dois anos, retornando com níveis pressóricos descontrolados $(260 \mathrm{x}$ $140 \mathrm{mmHg}$ ) e piora acentuada da função renal, detectada por elevação significativa das escórias (uréia $=160 \mathrm{mg} / \mathrm{dl}$, e creatinina $=5,9 \mathrm{mg} / \mathrm{dl}$ ). A mãe relatou que, durante o período de ausência de controle clínico, houve uso irregular da medicação e descontrole dos níveis pressóricos, necessitando de internações hospitalares. Nessa ocasião, foi novamente internado no HC- UFMG, onde permaneceu por uma semana e necessitou de associação de vários hipotensores em doses elevadas: nifedipina retard $-120 \mathrm{mg} / \mathrm{dia}$, captopril - $150 \mathrm{mg} / \mathrm{dia}$, triamtereno - $300 \mathrm{mg} / \mathrm{dia}$, hidroclorotiazida $-300 \mathrm{mg} /$ dia e minoxidil - $15 \mathrm{mg} /$ dia. Além do controle pressórico, foi iniciado tratamento conservador para disfunção renal, sobretudo pelo ajuste da dieta. Após essas medidas, o paciente encontra-se atualmente com PA controlada ( $130 \times 85 \mathrm{mmHg}$ ) pelo esquema citado e níveis de uréia em torno de $168 \mathrm{mg} / \mathrm{dl}$ e creatinina de $5,2 \mathrm{mg} / \mathrm{dl}$. A avaliação laboratorial durante o seguimento pode ser observada na Tabela 2.

\section{Discussão}

Alguns aspectos devem ser ressaltados em relação ao caso relatado. Inicialmente, chama atenção o paciente não ter sua pressão arterial medida até os treze anos de idade.
Isso levou a um diagnóstico tardio, com todas as implicações clínicas decorrentes de uma elevação crônica e acentuada dos níveis pressóricos. É interessante observar que os casos relatados na literatura tiveram diagnóstico estabelecido em diferentes faixas etárias ${ }^{3-7}$, variando de 10 meses $^{6}$ a 72 anos de idade ${ }^{4}$.

A principal alteração clínica observada nos pacientes com síndrome de Liddle é a hipertensão arterial, que pode ser explicada inicialmente pela expansão do fluido extracelular secundária à retenção renal de sódio. Esse processo produz diminuição dos níveis circulantes de renina, Ang I, Ang II e, conseqüentemente, de aldosterona ${ }^{3,4}$. No presente caso, apenas não houve diminuição de Ang I, como pode ser observado na Tabela 1. Além disso, esse aumento da pressão arterial é mantido às custas de uma elevação da resistência vascular periférica, atribuída ao maior influxo de sódio para a célula, com mobilização do cálcio intracelular e conseqüente vasoconstricção ${ }^{3,8}$. Observa-se, então, uma anormalidade nos mecanismos de transporte de sódio transmembrana, já demonstrada em estudos utilizando eritrócitos, sendo responsável pelo aumento da reatividade vascular ${ }^{8}$.

À semelhança deste caso, os pacientes com Síndrome de Liddle também exibem hipocalemia e retardo do crescimento $^{3-7}$. Do ponto de vista laboratorial, observa-se uma elevação de excreção urinária de potássio acoplada à retenção de sódio (Tabela 2). Essas alterações são similares no hiperaldosteronismo primário. A diferença básica, no entanto, diz respeito aos níveis circulantes de aldosterona, que se encontram reduzidos nesta doença, sendo classificada como pseudo-aldosteronismo ${ }^{1,2,7}$. Além da determinação da aldosterona, níveis reduzidos de ARP associados a

Tabela 2 - Exames laboratoriais ao diagnóstico e durante o acompanhamento clínico do paciente

\begin{tabular}{|c|c|c|c|c|}
\hline Exame/ Data & $02 / 97$ & $01 / 98$ & $04 / 00$ & 09/ 01 \\
\hline Uréia (VR:15-40) & $55 \mathrm{mg} / \mathrm{dl}$ & $71 \mathrm{mg} / \mathrm{dl}$ & $74 \mathrm{mg} / \mathrm{dl}$ & $168 \mathrm{mg} / \mathrm{dl}$ \\
\hline Creatinina (VR:0,5-1,0) & $1,1 \mathrm{mg} / \mathrm{dl}$ & $1,2 \mathrm{mg} / \mathrm{dl}$ & $1,9 \mathrm{mg} / \mathrm{dl}$ & $5,2 \mathrm{mg} / \mathrm{dl}$ \\
\hline $\mathrm{Na}^{+}(\mathrm{VR}: 135-145)$ & $129 \mathrm{mEq} / \mathrm{L}$ & $143 \mathrm{mEq} / \mathrm{L}$ & - & $139 \mathrm{mEq} / \mathrm{L}$ \\
\hline $\mathrm{K}^{+}(\mathrm{VR}: 3,5-5,5)$ & $2,2 \mathrm{mEq} / \mathrm{L}$ & $2,7 \mathrm{mEq} / \mathrm{L}$ & $2,9 \mathrm{mEq} / \mathrm{L}$ & $2,7 \mathrm{mEq} / \mathrm{L}$ \\
\hline $\mathrm{Cl}^{-}$(VR: 98-106) & $97 \mathrm{mmol} / \mathrm{l}$ & $96 \mathrm{mmol} / 1$ & $97 \mathrm{mmol} / 1$ & $96 \mathrm{mmol} / \mathrm{l}$ \\
\hline pH (VR: 7,35-7,45) & 7,42 & 7,46 & 7,33 & 7,34 \\
\hline $\mathrm{HCO}_{3}^{-}(\mathrm{VR}: 22-26)$ & 33 & 31,7 & 30,3 & 27,5 \\
\hline BE (VR: $-2 \mathrm{a}+2)$ & +9 & +7.6 & +3.1 & +1.5 \\
\hline Volume urinário & $2.900 \mathrm{ml}$ & $2.550 \mathrm{ml}$ & $3.200 \mathrm{ml}$ & - \\
\hline ClCr (VR: 88-166) & $34,9 \mathrm{ml} / \mathrm{min}$ & $38,2 \mathrm{ml} / \mathrm{min}$ & $31 \mathrm{ml} / \mathrm{min}$ & - \\
\hline $\mathrm{FE} \mathrm{Na}^{+}(\mathrm{VR}<1 \%)$ & $0,4 \%$ & $2,6 \%$ & - & - \\
\hline $\mathrm{FE} \mathrm{K}^{+}(\mathrm{VR}: 14,5 \pm 8,9)$ & $94 \%$ & $73,7 \%$ & - & - \\
\hline Proteinúria (VR: 1-15) & $130 \mathrm{mg} / \mathrm{dl}$ & & $128 \mathrm{mg} / \mathrm{dl}$ & - \\
\hline
\end{tabular}

VR: valor de referência, CICr: clearance de creatinina, FE: fração de excreção. 
cortisol circulante, dentro dos limites da normalidade, auxiliam na suspeita diagnóstica, apesar de levantarem outras possibilidades ${ }^{9}$. Nesse sentido, devem ser considerados, além de Síndrome de Liddle, o aldosteronismo remediável por glicocorticóides (GRA) e a síndrome de excesso aparente de mineralocorticóides (AME) $)^{9,10}$.

Todas essas três entidades cursam com níveis reduzidos de ARP ${ }^{9,10}$. O diagnóstico de certeza é dado pelo estudo genético dos pacientes ${ }^{2}$ ou pela dosagem dos metabólitos urinários do cortisol. No entanto, tais exames ainda são pouco disponíveis em nosso meio. Alguns achados clínicos e laboratoriais podem ser sugestivos de uma dessas enfermidades. O GRA é uma forma rara de hiperaldosteronismo, autossômica dominante, que ocorre devido a uma resposta anômala da zona glomerulosa da adrenal ao hormônio adrenocorticotrófico (ACTH) endógeno ${ }^{10}$. Nesses pacientes, o ACTH estimula de forma anômala a síntese de aldosterona, produzindo aumento de seus níveis circulantes, hipertensão arterial, hipocalemia e diminuição da ARP. A AME, por sua vez, caracteriza-se por uma alteração do receptor de aldosterona e do metabolismo do cortisol nas células tubulares renais. Tal defeito produz diminuição da degradação do cortisol e prolongamento de sua meia-vida prolongada, determinando uma atividade mineralocorticóidea anômala ${ }^{2}$. Dessa forma, os pacientes apresentam quadro clínico de hiperaldosteronismo, porém com níveis circulantes de aldosterona normais ou levemente diminuí$\operatorname{dos}^{2}$. O diagnóstico pode ser feito detectando-se excreção urinária anormal de metabólitos do cortisol. Tanto no GRA quanto na AME, o tratamento deve ser feito com glicocorticóides (dexametasona) associado a antagonistas de aldosterona, tipo espironolactona ${ }^{9,10}$. Nessas enfermidades, não há resposta satisfatória ao uso de triamtereno. No presente caso, a supressão acentuada dos níveis de aldosterona, associada à ótima resposta ao uso de triamtereno, reforçaram o diagnóstico de Síndrome de Liddle, tornando improváveis as duas outras entidades 5 .

Os estudos de linkage têm mostrado que o gene responsável pela doença encontra-se em um pequeno segmento do cromossoma 16. As mutações nesse gene produzem alterações nas subunidades beta e gama dos canais epiteliais de sódio, afetando sítios regulatórios e produzindo aumento de transporte iônico. Ocorre, então, uma ativação contínua desses canais, sem mecanismo de feedback ${ }^{2,3}$. Embora do ponto de vista genético a doença seja aparentemente similar entre os membros de uma mesma família, há diferentes expressões fenotípicas ${ }^{2,3}$. Vale destacar que, embora a genética tenha contribuído para o entendimento desta entidade, este tipo de análise não se encontra facilmente disponível em nosso meio. Apesar de útil, nem sempre é essencial ao diagnóstico, como ilustra o caso aqui relatado.

A resposta do paciente ao uso de triamtereno é característica da Síndrome de Liddle, e se explica pela ação direta da droga nos canais de sódio, diminuindo a reabsorção excessiva desse íon e, conseqüentemente, controlando a hipertensão e a hipocalemia ${ }^{5,6}$. A doença não envolve estímulos hormonais, diferindo do GRA e AME. No GRA, ocorre um aumento de aldosterona em resposta a um estímulo anômalo pelo $\mathrm{ACTH}$, com boa resposta tanto à administração de dexametasona quanto ao uso de espironolactona. No AME, também há resposta ao antagonismo dos receptores de aldosterona ${ }^{10}$.

A evolução deste paciente foi atípica quando comparada aos casos descritos da doença ${ }^{3-7}$. Em geral, não se observa perda da função renal. No entanto, o diagnóstico tardio, a má adesão ao tratamento e o descontrole dos níveis pressóricos provavelmente contribuíram para a progressão do dano renal. Um curso clínico similar foi relatado por Botero-Velez e colaboradores em uma paciente que necessitou de transplante renal aos 16 anos de idade ${ }^{3}$.

Finalmente, o presente relato ilustra claramente a importância da mensuração da pressão arterial rotineiramente em crianças. Este dado pode ser a pista inicial para o diagnóstico de doenças que exigem tratamento específico e aconselhamento genético, tais como a Síndrome de Liddle.

\section{Referências bibliográficas}

1. Liddle GW, Bledsoe T, Coppage WS. A familial renal disorder simulating primary aldosteronism but with negligible aldosterone secretion. Trans Assoc Am Physicians 1963; 76:199-213.

2. Warnock DG. Liddle syndrome: an autossomal dominant form of human hypertension. Kidney Int 1998; 53:18-24.

3. Botero-Velez M, Curtis JJ, Warnock DG. Brief report: Liddle's syndrome revised - a disorder of sodium reabsorption in the distal tubule. N Engl J Med 1994; 330:178-81.

4. Nakada T, Koike H, Akika T, Katayama T, Kawamata S, Takaya $\mathrm{K}$, et al. Liddle's syndrome, an uncommon form of hyporeninemic hypoaldosteronism: functional and histopathological studies. J Urol 1987; 137:636-40.

5. Wang C, Chan TK, Yeung RTT, Coghlan JP, Scoggins BA, Stockigt JR. The effect of triamterene and sodium intake on renin, aldosterone, and erythrocyte sodium transport in Liddle's syndrome. J Clin Endocrinol Metab 1981; 52:1027-32.

6. Vania A, Tucciarone L, Mazzeo D, Capodaglio PF, Cugini P. Liddle's syndrome: a 14-year follow-up of the youngest diagnosed case. Pediatr Nephrol 1997; 11:7-11.

7. Hyman PE, Shalafi RI, Tan SY, Hintz R. Liddle syndrome. J Pediatr 1979; 95:77-8.

8. Gardner JD, Lapey A, Simopoulos AP, Bravo EL. Abnormal membrane sodium transport in Liddle's syndrome. J Clin Invest 1971; 50:2253-8.

9. Yiu VWY, Dluhy RG, Lifton RP, Guay-Woodford LM. Low peripheral plasma renin activity as a critical marker in pediatric hypertension. Pediatr Nephrol 1997; 11:343-6.

10. Warnock DG. Low renin hypertension in the next millennium. Semin Nephrol 2000; 20:40-6.

Endereço para correspondência:

Dr. Eduardo A. Oliveira

Rua Patagônia, 515 / 701

CEP 30320-080 - Belo Horizonte, MG

E-mail: eduolive@medicina.ufmg.br 